\title{
Pharmacologic Treatment of Complex Regional Pain Syndrome I: A Conceptual Framework
}

\author{
Gerard M. Ribbers, MD, PhD, Alexander C. Geurts, MD, PhD, Henk J. Stam, MD, PhD, Theo Mulder, PhD
}

ABSTRACT. Ribbers GM, Geurts AC, Stam HJ, Mulder T. Pharmacologic treatment of complex regional pain syndrome I: a conceptual framework. Arch Phys Med Rehabil 2003;84: 141-6.

Pain may be a leading symptom in complex regional pain syndrome type I (CRPS I) and may hinder functional recovery. In this case, a pharmacotherapeutic approach to pain should be part of the individually tailored interdisciplinary treatment regimen. However, operational criteria for determining which patient may profit from what therapeutic intervention are lacking. This article discusses a conceptual framework in which the rapid progress made in basic pain research may contribute to the clinical management of pain in CRPS I. First, recent insights in the pathophysiologic mechanisms underlying CRPS I are reviewed. CRPS I is considered a neuropathic pain syndrome with a mixed and time-dependent profile of a regional inflammation, sensitization of primary somatosensory afferents (peripheral sensitization), and sensitization of spinal neurons (central sensitization). The dominant mechanisms may vary across individual patients with different time profiles. Second, a model was constructed in which signs and symptoms in an individual patient are related to these mechanisms. Finally, relating the clinical picture to the underlying pathophysiology may help determine the pharmacotherapeutic approach for an individual patient. Pharmacologic options are discussed in this context. The presented framework does not aim to provide an evidence-based treatment algorithm, ready to be used in daily clinical practice; rather it offers a crude, first step toward a mechanism-based pharmacotherapy in CRPS I, in an effort to shift from a mainly empirical treatment paradigm toward theory-driven treatment procedures.

Key Words: Complex regional pain syndrome, type I; Pharmacotherapy; Reflex sympathetic dystrophy; Rehabilitation; Treatment outcome.

(C) 2003 by the American Congress of Rehabilitation Medicine and the American Academy of Physical Medicine and Rehabilitation

$\mathbf{R}$ EFLEX SYMPATHETIC DYSTROPHY is a pain syndrome associated with vasomotor and sudomotor abnormalities. Numerous names have been attributed to this syndrome, all sharing a lack of formal, standardized diagnostic criteria. In response to this problem, the International Associ-

From the Rehabilitation Center Rijndam (Ribbers) and Institute of Rehabilitation (Ribbers, Stam), Erasmus Medical Center, Rotterdam; Sint Maartenskliniek-Research and Department of Rehabilitation Medicine, Sint Maartenskliniek, Nijmegen (Geurts); and Institute of Human Movement Sciences, University of Groningen, Groningen (Mulder), the Netherlands.

No commercial party having a direct financial interest in the results of the research supporting this article has or will confer a benefit upon the author(s) or upon any organization with which the author(s) is/are associated.

Reprint requests to Gerard M. Ribbers, MD, PhD, Rehabilitation Center Rijndam, PB 23181, 3001 KD Rotterdam, The Netherlands, e-mail: g.ribbers@rra.nl.

0003-9993/03/8401-6770\$35.00/0

doi:10.1053/apmr.2003.50076 ation for the Study of Pain (IASP) in 1994 introduced the term complex regional pain syndrome type I (CRPS I) (table 1$).{ }^{1}$

A well-accepted treatment algorithm for CRPS I is lacking. Treatment regimens for CRPS I vary widely and may include physical therapy, sympathetic nerve blocks, tricyclic antidepressants (TCAs), opiates, anticonvulsives, and psychologic treatment. ${ }^{2}$ In a consensus meeting of leading experts on CRPS I, IASP aimed to counterbalance this heterogeneous approach. It was stated that functional recovery through a measured pace should at all times be the ultimate goal of treatment. ${ }^{3}$ Initially, desensitization and overcoming fear of movement is considered important, allowing the limbs to be touched and the patient to start moving. This phase should be followed by isometric strengthening, stress loading, and general aerobic conditioning. The last step should be aimed at complete functional recovery. Psychologic management may be necessary in the case of overprotection, fear of movement, irrational inferences, or psychiatric problems such as depression. It was also stated that if pain is a limiting factor in achieving progress, pharmacologic treatment is indicated. However, guidelines for the choice of pharmacotherapy in CRPS I are lacking.

The present article therefore describes a conceptual framework in which recent insights into the mechanisms underlying pain in CRPS I are discussed and related to pharmacologic treatment. First, CRPS I is discussed in the context of progress made in basic research on neuropathic pain. Second, how the clinical assessment of the individual patient may refer to the underlying mechanisms is discussed. Finally, a model is constructed in which the signs and symptoms of an individual patient may help target pharmacotherapy to underlying mechanisms.

\section{CRPS I: A NEUROPATHIC PAIN SYNDROME}

Pain is a leading symptom in CRPS I. However, its actual cause remains obscure, and its treatment is underevaluated. ${ }^{4}$ By its definition, neuropathic pain is initiated or caused by a primary lesion or dysfunction in the nervous system and, as such, pain in CRPS I may be considered as of neuropathic origin. ${ }^{1}$ In neuropathic pain, the relation between the etiology of pain and presenting symptoms is very complex. Diseases of different etiologies may have common pain mechanisms, a single mechanism may explain different symptoms, and, furthermore, a similar symptom in separate patients may be caused by different mechanisms. ${ }^{5,6}$

Normal sensory function depends on a critical equilibrium between neurons and their environment, and a disruption of this equilibrium may initiate profound changes in sensory function. Functional alterations in the complex interactions between neurons and their environment seem to play a pivotal role in CRPS I, and basic research focuses on mechanisms such as peripheral and central sensitization of somatosensory afferents and the influence of the sympathetic nervous system on them. These mechanisms are reviewed briefly later.

\section{Peripheral Sensitization}

Noxious messages arise from free unmyelinated terminal arborizations in cutaneous, muscular, and joint tissues. ${ }^{7}$ Some 
Table 1: IASP Criteria for CRPS I

1. The presence of an initiating noxious event, or a cause of immobilization.

2. Continuing pain, allodynia, or hyperalgesia in which the pain is disproportionate to any inciting event.

3. Evidence at some time of edema, changes in skin blood flow, or abnormal sudomotor activity in the region of pain.

4. This diagnosis is excluded by the existence of conditions that would otherwise account for the degree of pain and dysfunction.

NOTE: When the criteria 2 through 4 have to be satisfied, this is not the case for the presence of an initiating event. Atrophy (of hair, nails, and other soft tissues), alterations in hair growth, loss of joint mobility, impairment of motor function, and sympathetically maintained pain may be associated signs and symptoms but are not used for diagnosis.

of these fibers specifically act as nociceptors, and various types have been identified, such as polymodal nociceptors (responsive to thermal, mechanical, and chemical stimuli), A $\delta$ mechanothermal nociceptors, and high-threshold $\mathrm{A} \delta$-mechanoreceptors. Other fibers are not specific nociceptors. They are activated by nonnoxious stimuli, with increasing activity as the intensity of the stimulus increases. The sensitivity of this complex and heteromodal peripheral terminal of afferents is not fixed. ${ }^{8}$ Under pathologic conditions, such as inflammation, the responsiveness of the primary afferents may increase (peripheral sensitization). Indeed, an excessive inflammatory response in CRPS I has been suggested. ${ }^{9,10}$ A variety of chemical mediators, present in the "inflammatory soup" surrounding the primary afferents, have been identified. ${ }^{11}$ These mediators are of vascular origin or are produced by the damaged tissue, afferent fibers themselves, sympathetic fibers, and/or various immune cells.

The reactive oxygen species-such as hydrogen peroxide, superoxide, and hydroxyl species - may cause oxidative stress and enhance the effects of other inflammatory mediators, such as bradykinin and prostaglandin. ${ }^{12}$ Nitrous oxide, another reactive molecule with proinflammatory actions, may contribute to ectopic discharges in primary afferents. These and many other mediators (eg, protons, kinins, prostanoids, serotonin, histamine, adenosine triphosphate) take part in a series of responses that cause changes in local blood flow, vascular permeability, activation and migration of immune cells, as well as in changes in the release of growth and trophic factors from surrounding tissue. These processes induce a state of hyperexcitability, mobilizing local defense mechanisms and contributing to the prevention of (re)traumatization. Several ion channels are involved in peripheral sensitization. Sodium channels, especially the ones insensitive to tetradotoxin, calcium channels, and the capsaicin or vanilloid receptor, are the most prominent ones. ${ }^{13-15}$

\section{Central Sensitization}

In addition to impulse generation and propagation, $\mathrm{C}$ fibers display axonal transport of chemicals such as glutamate and substance $\mathrm{P}$. This so-called chemical signaling causes an increased responsiveness of dorsal horn neurons to all forms of input (central sensitization). The $N$-methyl-D-aspartate (NMDA) receptor is especially important in this process. ${ }^{16-18} \mathrm{~A}$ change in phenotype and anatomy of afferents may also contribute to the increased sensitivity of spinal cord neurons. ${ }^{19}$ In the case of inflammation, A beta fibers start expressing substance $\mathrm{P}$ and calcitonine gene-related peptide. These transmit- ters are involved in central sensitization and are normally only produced by $\mathrm{C}$ and $\mathrm{A}$ delta fibers in transmitting nociceptive signals. Via this route, nonnoxious stimuli that activate A beta fibers may start contributing to central sensitization. Furthermore, sprouted A beta fibers invade lamina II where they interact with neurons that normally receive nociceptor input via $\mathrm{C}$ fibers. The sprouting causes lamina II to start receiving nonnoxious stimuli, offering an anatomic substrate in which nonnoxious stimuli may be misinterpreted as noxious.

\section{Sympathetically Maintained Pain}

For many years, pain relief after sympathectomy was considered pathognomonic for CRPS I; sympathectomies have been used as diagnostic tests and were considered first-choice treatment. ${ }^{20}$ Today, the pivotal role of sympathetic vasoconstrictor hyperactivity in regional vasomotor and sudomotor abnormalities in CRPS I is questioned. ${ }^{21,22}$ Nevertheless, the contribution of the sympathetic nervous system to CRPS I remains undetermined. In neuropathic pain, activity in sympathetic neurons may cause excitation of primary afferents, generating pain. This phenomenon is known as sympathetically maintained pain (SMP). However, especially in more chronic stages, sympathectomy may fail to relieve pain (sympathetically independent pain [SIP]). ${ }^{23}$ Almost any type of neuropathic pain disorder, such as phantom pain, metabolic neuropathies, herpes zoster, and CRPS I, may manifest with elements of SMP or SIP. Pain may be mixed SMP and SIP, and the relative contribution of SMP may vary over time.

Animal models with partially lesioned nerves have revealed several mechanisms that may explain how sympathetic discharge may lead to neuropathic pain. These include the sprouting of sympathetic afferents to the axons and dorsal root ganglia of the primary afferents (basket formation) and the expression of $\alpha$ adrenoreceptors on injured and uninjured axons. ${ }^{24}$ Sympathetic outflow also triggers depolarization of uninjured $\mathrm{C}$ fibers, however, only when these afferents are embedded in an inflamed skin. ${ }^{25,26}$ Furthermore, activated $\alpha_{2}$ adrenergic autoreceptors on the sympathetic terminal evoke the synthesis of prostaglandin, which is involved in the sensitization of the $\mathrm{C}$ afferents. ${ }^{27,28}$ The responsiveness of $\mathrm{C}$ afferents to sympathetic outflow is to be considered physiologic and should subside when the inflammation subsides. The pathology (SMP) appears when the inflammation persists or when the adrenergic responsiveness does not disappear. ${ }^{29}$

\section{HOW THE SIGNS AND SYMPTOMS OF NEUROPATHIC PAIN RELATE TO THE MECHANISMS}

As discussed, multiple mechanisms underlying pain may operate simultaneously or on different time scales. It is the clinician's challenge to identify the mechanisms operating in an individual patient and to target treatment for them. Woolf and Decosterd ${ }^{5}$ and Woolf and Mannion ${ }^{6}$ have formulated a preliminary framework to achieve this.

\section{Stimulus-Independent Pain}

A regional inflammatory reaction may cause functional and even phenotypical changes in primary afferents, resulting in altered discharge patterns. Spontaneous depolarization of primary afferents, independent of any sensory stimulus, is a key characteristic of peripheral sensitization. ${ }^{6}$ In $\mathrm{C}$ fibers, this may cause persistent burning pain, in A delta afferents a sharp lancinating pain, and in A beta fibers-which normally signal nonnoxious stimuli-a dysesthetic tingling sensation.

As discussed earlier, the spontaneous activity of sensory neurons, as a key characteristic of peripheral sensitization, may 
Table 2: Mechanism-Based Pharmacotherapy in CRPS I

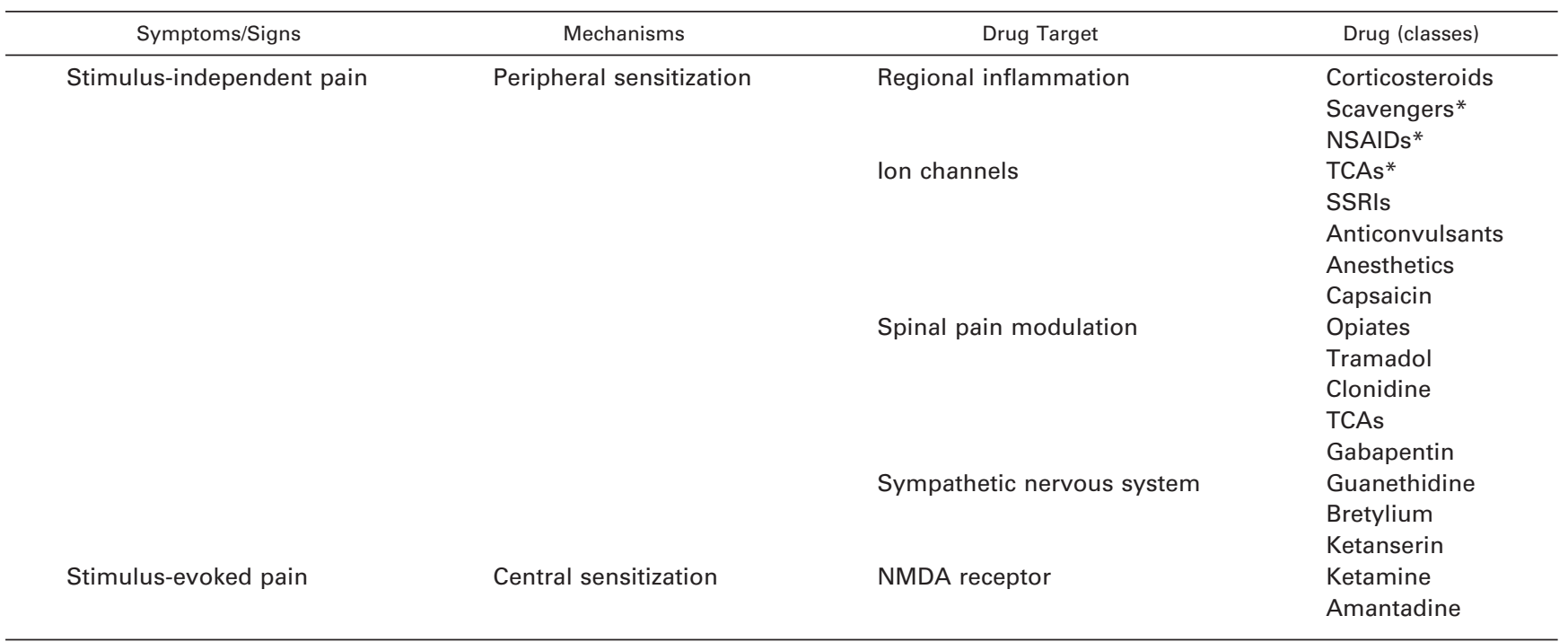

Abbreviations: NSAIDs, nonsteroidal anti-inflammatory drugs; SSRIs, serotonin-selective reuptake inhibitors.

* See text.

depend on several mechanisms ${ }^{11-15}$ : (1) an accumulation or activation of specific ion channels (sodium, calcium, vanilloid receptor), (2) SMP may be a component of stimulus-independent pain, and (3) decreased inhibitory input at a spinal level. Descending control of pain is manifested via pathways that originate at the level of the cortex, the thalamus, and the brainstem. ${ }^{30}$ Serotonin, noradrenaline, $\gamma$-aminobutyric acid (GABA), and the endogenous opioids are the main transmitters that are involved in the descending modulation of pain.

\section{Stimulus-Evoked Pain}

In physiologic conditions, pain is only elicited by intense mechanical, thermal, or chemical stimuli. In pathologic conditions, a state of an exaggerated sensitivity to mechanical, thermal, or chemical stimuli occurs with 2 key features: hyperalgesia and allodynia. ${ }^{6}$ Hyperalgesia is an increased pain response to what would normally be a suprathreshold noxious stimulus, and results from abnormal processing of nociceptor input. Allodynia is a pain response caused by what would normally be a nonnoxious stimulus. Allodynia can be caused both by decreased thresholds of peripheral $\mathrm{C}$ afferents, as in peripheral sensitization, and by a state of hyperexcitability in the dorsal horn of the spinal cord, as in central sensitization. Because these 2 different causes of allodynia cannot be distinguished clinically, assessing allodynia does not help to target treatment to peripheral or central sensitization. Hyperalgesia, on the other hand, represents an NMDA-mediated state of hyperexcitability with increased responses of dorsal horn neurons to peripheral stimuli (central sensitization). It is characterized by tactile or dynamic hyperalgesia, cold hyperalgesia, and pinprick hyperalgesia, and may manifest in 3 ways: enlargement of the area in the periphery where a stimulus will activate neurons, increased responses to suprathreshold stimuli, and previously subthreshold inputs that initiate action potentials.

\section{CLINICAL IMPLICATIONS}

In CRPS I, a prolonged regional inflammation may induce sensitization of primary and secondary somatosensory afferents (peripheral and central sensitization). These aspects of neuro- pathic pain may be present in each patient in a unique individual mix and with different time profiles. Both the sensitization of the somatosensory afferents as well as the regional inflammatory reaction are determined by mechanisms that add together and have only partially been elucidated. To improve the efficacy of pharmacologic treatment in CRPS I, effort should be made in aiming treatment at the dominant mechanisms underlying the regional inflammation and peripheral and/or central sensitization in an individual patient at a specific moment in time. Although most available drugs lack the specificity to fit in this model of mechanism-based prescription, drug classes that are commonly used in neuropathic pain are discussed to show the concept (table 2).

\section{Regional Inflammatory Reaction}

The presence of neuroimmune interactions in neuropathic pain suggests responsiveness to immunosuppressive therapy. Indeed, there is some preclinical evidence with cyclosporine, thalidomide, and methotrexate to support this. ${ }^{29,31}$ Immunosuppressives are not used in the clinical management of neuropathic pain. Drugs that are commonly used to treat the inflammatory symptoms of CRPS I are nonsteroidal anti-inflammatory drugs (NSAIDs), corticosteroids, and free-radical scavengers.

Corticosteroids are known to relieve neuropathic pain in animal models. ${ }^{29}$ In a recent review on controlled clinical trials in CRPS, Kingery ${ }^{32}$ showed that oral corticosteroids are the only drug effective with long-term results. A similar result was obtained in a systematic review ${ }^{33}$ on treatment of poststroke shoulder hand syndrome, often associated with CRPS I. Reactive oxygen species are known to be involved in inflammatory processes and are suggested to be involved in CRPS I. Welldesigned studies ${ }^{34,35}$ on the scavengers dimethyl sulphoxide (DMSO) and vitamin C support this hypothesis. In a prospective, randomized and double-blind study, ${ }^{34} 16$ patients treated with 50\% DMSO were compared with 15 patients treated only with an indifferent fatty cream. The group treated with $50 \%$ DMSO exhibited significantly reduced inflammatory symptoms in CRPS I without a significant pain relief. In another randomized clinical trial, ${ }^{35} 52$ patients with 54 wrist fractures 
were treated with vitamin $\mathrm{C}$ and compared with 63 patients with 65 wrist fractures treated with placebo. CRPS I occurred in $7.4 \%$ of the patients treated with vitamin $\mathrm{C}$ and in $21.5 \%$ of those treated with placebo. These studies may seem promising, but should be repeated with larger numbers of patients.

NSAIDs share the ability to inhibit the cyclooxygenasemediated synthesis of prostaglandins, important mediators of inflammatory hyperalgesia. Besides the peripheral anti-inflammatory action of NSAIDs, an analgesic action by blocking spinal nociceptive processing has been emphasized. ${ }^{11,36}$ However, the results of NSAIDs in several neuropathic pain trials are mixed, and well-designed studies in CRPS I are lacking. In a study by Rico et al, ${ }^{37}$ NSAIDs failed to show any effect in CRPS I. Nevertheless, the IASP consensus report states that NSAIDs should be considered, especially when treating the early manifestation of the inflammatory signs of CRPS I. ${ }^{3}$

\section{Stimulus-Independent Pain}

When pain is stimulus independent, treatment should be aimed at the mechanisms underlying the sensitization of the primary somatosensory afferents. Ion channel blockers, sympathectomy, or drugs that facilitate spinal inhibitory mechanisms, such as opiates and clonidine, may be used ${ }^{38}$ (table 2).

Ion channel blockers may be considered the drug treatment of choice in cases of stimulus-independent pain. TCAs are the first drugs proven effective in neuropathic pain. ${ }^{39}$ Sindrup and Jenssen ${ }^{40}$ have calculated that TCAs, which cause a balanced reuptake inhibition of both serotonin and noradrenaline (imipramine, amitriptyline, clomipramine), have a number needed to treat of $2.0(1.7-2.5)$ in painful polyneuropathy. This means that if 2 patients are treated, 1 will experience at least $50 \%$ relief of pain. The relative selective noradrenaline reuptake blockers desipramine and clomipramine seem less effective, with a number needed to treat of 3.4 (2.3-6.6). The efficacy of TCAs in treating neuropathic pain may even be enhanced if they are not dosed according to effects and side effects but to optimal plasma levels. However, TCAs are also well known because of their bothersome side-effect profile. The newer antidepressants, the serotonin-selective reuptake inhibitors (SSRIs), have a more favorable side-effect profile. However, SSRIs seem less effective as analgesics in neuropathic pain, with a number needed to treat of 2.9 (paroxetine) and 7.7 (citalopram). ${ }^{40}$ Carbamazepine and phenytoin exert a sodium channel blocking effect, reducing the excitability of sensitized $\mathrm{C}$ fibers. In studies on diabetic polyneuropathy, numbers needed to treat of 3.3 (carbamazepine) and 2.1 (phenytoin) have been calculated. The novel anticonvulsant gabapentin has a more favorable side-effect profile than carbamazepine and blocks the voltage gated calcium channel in the terminals of the primary afferents. ${ }^{14,41}$ Its effectiveness in CRPS I has been described in a case study. ${ }^{42}$ In a randomized controlled trial ${ }^{43}$ on painful diabetic polyneuropathy with 165 patients, a number needed to treat of $3.7(2.4-8.3)$ was reported.

Other ion channel blockers such as mexiletine (the oral analog of lidocaine), lidocaine patches, or capsaicin are used in treating neuropathic pain, but their efficacy has not been convincingly shown. ${ }^{40,44}$

Pain in CRPS I may be SMP. However, sympathectomy in CRPS I remains controversial. Kingery's meta-analysis ${ }^{32}$ showed poor outcome with sympathectomies. Alternatives to regional intravenous treatments with substances that mimic guanethidine, such bretylium and ketanserin, have been reported effective in single studies but have not been confirmed. ${ }^{45,46}$ It has been suggested that the intravenous administration of phentolamine, an $\alpha$-adrenergic blocking agent, may predict whether an SMP component is present in CRPS I and thus if sympathectomy should be considered. ${ }^{47}$ Nevertheless, despite the fact it has been applied for nearly a century, the scientific support for performing sympathetectomy in CRPS I remains weak.

Reduced inhibitory inputs, from the spinal cord or descending from the brain, may also be involved in stimulus-independent pain. Opiates, gabapentin, TCAs, GABA-enhancing drugs, or drugs such as clonidine that mimic descending inhibition may be useful in augmenting central inhibition. ${ }^{48}$ Clonidine has been administered transdermally with patches in diabetic polyneuropathy, orally in postherpetic neuralgia, intrathecally in neuropathic rats, and its use has been described in a case study in CRPS I. ${ }^{49-51}$ However, its effectiveness has still not been convincingly shown. ${ }^{32}$ Effective long-term treatment with intrathecal morphine alone has been reported in 2 cases of CRPS I. ${ }^{52}$ Orally administered oxycodone in combination with antidepressants has been tested in postherpetic neuralgia, with a number needed to treat of $2.5(1.6-5.1) .40$ Tramadol may be an alternative to strong opioids because of its favorable sideeffect profile, with a number needed to treat of $3.4(2.3-6.4)$ in painful polyneuropathy. However, prescribing opioids in neuropathic pain remains controversial and should be considered as the last option. ${ }^{32,53}$

\section{Stimulus-Evoked Pain}

When pain is stimulus evoked, treatment should be aimed at central sensitization. NMDA receptors are involved in central sensitization, and several studies report on the analgesic effects of clinically available NMDA receptor antagonists, such as ketamine and amantadine. ${ }^{17,18,54,55}$ However, convincing controlled trials are lacking, and NMDA antagonists are known for their side effects such as hallucinations, vivid dreaming, and auditory and visual disturbances. The side-effect profile of amantadine is better than ketamine, and long-term effects of amantadine, lasting beyond the drug presence in body tissues, have been described. ${ }^{17,56}$

\section{CONCLUSION}

Pharmacotherapy may be part of an individually tailored multidisciplinary treatment regimen in CRPS I, when pain hinders functional progress. However, operational criteria to determine which patient may profit from what treatment are absent, and sound research data on the effectiveness of pharmacologic treatment in CRPS I are lacking. It is argued that pain in CRPS I may be considered as being of neuropathic origin. Basic pain research has shown that, irrespective of the underlying pathology, different mechanisms may underlie neuropathic pain (eg, accumulation of sodium channels or sensitization of NMDA receptors). Mechanism-based pharmacotherapy as promoted by Woolf and Decosterd ${ }^{5}$ and Woolf and Mannion $^{6}$ encompasses challenges for clinicians and for clinical and basic researchers. First, further insight into the mechanisms underlying pain should be accompanied by the development of new diagnostic tools enabling a more accurate categorization of pain, thereby increasing the efficiency of a mechanism-based pain assessment. Second, drugs should be developed with a high specificity for single mechanisms, allowing mechanism-based pharmacotherapy. Finally, within the context of evidence-based pain treatment instead of simple global pain measures such as visual analog scale scores, new clinical outcome measures referring to specific pain mechanisms should be developed.

The presented framework is incomplete and may serve only as a starting point for formulating a mechanism-based pharma- 
cologic treatment of CRPS I. However, if the gap between basic pain research and clinical pain management in CRPS I is to be bridged, the approach in which drug treatment is targeted to the underlying mechanisms seems necessary. This will need an intense and ongoing collaboration between clinicians and preclinical and clinical researchers.

\section{References}

1. Merskey H, Bogduk N. Classification of chronic pain. Seattle: IASP Pr; 1994.

2. Allen G, Galer B, Schwartz L. Epidemiology of complex regional pain syndrome: a retrospective chart review of 134 patients. Pain 1999;80:539-44

3. Stanton-Hicks M, Baron R, Boas R, et al. Complex regional pain syndromes: guidelines for therapy. Clin J Pain 1998;14:155-66.

4. Geertzen J. Reflex sympathetic dystrophy: a study in the perspective of rehabilitation medicine [thesis]. Groningen: Rijksuniversiteit Groningen; 1998.

5. Woolf CJ, Decosterd I. Implications of recent advances in the understanding of pain pathophysiology for the assessment of pain in patients. Pain 1999;Suppl 6:S141-7.

6. Woolf CJ, Mannion RJ. Neuropathic pain: aetiology, symptoms, mechanisms, and management. Lancet 1999;353:1959-64.

7. Belmonte C, Cervero F. Neurobiology of nociceptors. Oxford: Oxford Univ Pr; 1996.

8. Schaible H, Grubb BD. Afferent and spinal mechanisms of joint pain. Pain 1993;55:5-54

9. Oyen WJ, Arntz IE, Claessens RA, van der Meer JW, Corstens FH, Goris RA. Reflex sympathetic dystrophy of the hand: an inflammatory response? Pain 1993:55:151-7.

10. Calder JS, Holten I, McAllister RM. Evidence for immune system involvement in reflex sympathetic dystrophy. J Hand Surg [Br] $1998 ; 23: 147-50$

11. Dray A. Inflammatory mediators of pain. Br J Anaesth 1995;75: 125-31.

12. Goris RJ, Dongen LM, Winters HA. Are toxic free radicals involved in the pathogenesis of reflex sympathetic dystrophy? Free Radic Res Commun 1987;3:13-8.

13. Novakovic SD, Tzoumaka E, McGivern JG, et al. Distribution of the tetrodotoxin-resistant sodium channel PN3 in rat sensory neurons in normal and in neuropathic conditions. J Neurosci 1998; 18:2174-87.

14. Meder WP, Dooley DJ. Modulation of $\mathrm{K}(+)$ induced synaptosomal calcium influx by gabapentin. Brain Res 2000;875:157-9.

15. Winter J, Bevan S, Campbell EA. Capsaicin and pain mechanisms. Br J Anaesth 1995;75:157-68.

16. Attal N, Brasseur L, Chauvin M, Bouharissa D. A case of pure dynamic mechano-allodynia due to a lesion of the spinal cord: pathophysiological considerations. Pain 1998;75:399-404.

17. Eisenberg E, Pud D. Can patients with chronic neuropathic pain be cured by acute administration of the NMDA receptor antagonist amantadine? Pain 1998:74:337-9.

18. Takahashi H, Miyazaki M, Nanbu T, Yanagida H, Morita S. The NMDA-receptor antagonist ketamine abolishes neuropathic pain after epidural administration in a clinical case. Pain 1998;75: $391-4$.

19. Koerber HR, Mirnics K, Brown PB, Mendell LM. Central sprouting and functional plasticity of regenerated primary afferents. J Neurosci 1994;14:3655-71.

20. Schürmann M, Gradl G, Andress HJ, Fürst H, Schildberg FW. Assessment of peripheral sympathetic nervous function for diagnosing early post-traumatic complex regional pain syndrome type I. Pain 1999;80:149-59.

21. Schott GD. An unsympathetic view of pain. Lancet 1995;345: 634-6.

22. Drummond PD, Finch PM, Smythe GA. Reflex sympathetic dystrophy: the significance of differing plasma catecholamine concentrations in affected and unaffected limbs. Brain 1991;114: 2025-36.

23. Roberts WJ. A hypothesis of the physiological basis for causalgia and related pains. Pain 1986;24:279-311.
24. Ramer MS, Thompson SW, McMahon SB. Causes and consequences of sympathetic basket formation in dorsal root ganglia. Pain 1999;6:111-20.

25. Sato J, Perl ER. Adrenergic excitation of cutaneous pain receptors induced by peripheral nerve injury. Science 1991;251:1608-10.

26. Sato J, Suzuki S, Iseki T, Kumazawa T. Adrenergic excitation of cutaneous nociceptors in chronically inflamed rats. Neurosci Lett 1993;64:225-8

27. Levine JD, Taiwo YO, Collins SD, Tam JK. Noradrenaline hyperalgesia is mediated through interaction with sympathetic postganglionic neuron terminals rather than activation of primary afferent nociceptors. Nature 1986;323:158-60.

28. Gonzales R, Sherbourne CD, Goldyne ME, Levine JD. Noradrenaline-induced prostaglandin production by sympathetic postganglionic neurons is mediated alpha-2-adrenergic receptors. J Neurochem 1991;57:1145-50

29. Bennet GJ. Scientific basis for the evaluation and treatment of RSD/CRPS syndromes: laboratory studies in animals and man. In: Max M, editor. Pain 1999-an updated review. Seattle: IASP Pr; 1999. p 331-9.

30. Stamford JA. Descending control of pain. Br J Anaesth 1995;75: 217-27.

31. Hashizume H, Rutkowski MD, Weinstein JN, DeLeo JA. Central administration of methotrexate reduces mechanical allodynia in an animal modal of radiculopathy/sciata. Pain 2000;87:159-69.

32. Kingery WS. A critical review of controlled clinical trials for peripheral neuropathic pain and complex regional pain syndromes. Pain 1997;73:123-39.

33. Geurts AC, Visschers BA, van Limbeek J, Ribbers GM. Systematic review of aetiology and treatment of post-stroke hand edema and shoulder hand syndrome. Scand J Rehabil Med 2000;32:4-10.

34. Zuurmond WW, Langendijk PN, Bezemer PD, Brink HE, de Lange JJ, van Loenen AC. Treatment of acute reflex sympathetic dystrophy with DMSO 50\% in a fatty cream. Acta Anaesthesiol Scand 1996;40:364-7.

35. Zollinger PE, Tuinbebreijer WE, Kreis RW, Breederveld RS Effect of vitamin $\mathrm{C}$ on frequency of reflex sympathetic dystrophy in wrist fractures: a randomised trial. Lancet 1999;354:2025-8.

36. Geisslinger G, Yaksh TL. Spinal actions of cyclooxygenase isoenzyme inhibitors. In: Devor M, Rowbotham MC, Wiesenfeld-Halin Z, editors. Proceedings of the 9th World Congress on Pain. Seattle: IASP Pr; 2000. p 771-85.

37. Rico H, Merono E, Gomez-Castresana F, Torrubiano, Espinos D, Diaz P. Scintigraphic evaluation of reflex sympathetic dystrophy: comparative study of the course of the disease under two therapeutic regimens. Clin Rheumatol 1987;6:233-7.

38. Rowbotham MC, Petersen KL, Davies PS, Friedman EK, Fields HL. Recent developments in the treatment of neuropathic pain. In: Devor M, Rowbotham MC, Wiesenfeld-Halin Z, editors. Proceedings of the 9th World Congress on Pain. Seattle: IASP Pr; 2000. p 833-55.

39. Watson CP, Evans RJ, Reed K, Merskey H, Goldsmith L, Warsh J. Amitriptyline versus placebo in post herpetic neuralgia. Neurology 1982;32:671-3.

40. Sindrup SH, Jenssen TS. Efficacy of pharmacological treatments of neuropathic pain: an update and effect related to mechanism of drug action. Pain 1999;83:389-400.

41. Nicholson B. Gabapentin use in neuropathic pain syndromes. Acta Neurol Scand 2000;101:359-71.

42. Mellick GA, Mellick LB. Reflex sympathetic dystrophy treated with gabapentin. Arch Phys Med Rehabil 1997;78:98-105.

43. Backonja M, Beydoun A, Edwards KR, et al. Gabapentin for the symptomatic treatment of painful neuropathy in patients diabetes mellitus: a randomized controlled trial. JAMA 1998; 280:1831-6.

44. McQuay HJ, Moore RA, Ecclestone C, Morley S, Williams AC. Systematic review of outpatient services for chronic pain control. Health Technol Assess 1997;1:1-135.

45. Hord AH, Rooks MD, Stephens BO, Rogers HG, Fleming LL. Intravenous regional bretylium and lidocaine for treatment of reflex sympathetic dystrophy: a randomized, double-blind study. Anesth Analg 1992;74:818-21. 
46. Hanna MH, Peat SJ. Ketanserin in reflex sympathetic dystrophy. A double-blind placebo controlled cross-over trial. Pain 1989;38: 145-50.

47. Arnér S. Intravenous phentolamine test: diagnostic and prognostic use in reflex sympathetic dystrophy. Pain 1991;46:17-22.

48. Dickinson AH. Spinal cord pharmacology of pain. Br J Anaesth 1995;75:193-200.

49. Rauck RL, Eisenach JC, Jackson K, Young LD, Southern J. Epidural clonidine treatment for refractory reflex sympathetic dystrophy. Anesthesiology 1993;79:1163-9.

50. Pan HL, Chen SR, Eisenach JC. Intrathecal clonidine alleviates allodynia in neuropathic rats: interaction with spinal muscarinic and nicotinic receptors. Anesthesiology 1999;90:509-14.

51. Siddal PJ, Molloy AR, Walker S, Mather LE, Rutkowski SB, Cousins MJ. The efficacy of intrathecal morphine and clonidine in the treatment of pain after spinal cord injury. Anesth Analg 2000;91:1493-8
52. Becker WJ, Ablett DP, Harris CJ, Dold ON. Long term treatment of intractable reflex sympathetic dystrophy with intrathecal morphine. Can J Neurol Sci 1995;22:153-9.

53. Dellemijn P. Are opioids effective in relieving neuropathic pain? Pain 1999;80:453-62.

54. Mercadante S, Arcuri E, Tirelli W, Casuccio A. Analgesic effect of intravenous ketamine in cancer patients on morphine therapy. A randomized, controlled, double blind, crossover, double-dose study. J Pain Symptom Manage 2000;20:246-52.

55. Pud D, Eisenberg E, Spitzer A, Adler R, Gried G, Yarnitsky D. The NMDA receptor antagonist amantadine reduces surgical neuropathic pain in cancer patients: a double blind, randomized, placebo controlled trial. Pain 1998;75:349-54.

56. Eide PK. Clinical trials of NMDA receptor antagonists as analgesics. In: Devor M, Rowbotham MC, Wiesenfeld-Halin Z, editors. Proceedings of the 9th World Congress on Pain. Seattle: IASP Pr; 2000. p 817-32. 